\title{
Condições oftalmológicas de pacientes com síndrome da imunodeficiência adquirida com longo tempo de seguimento
}

\author{
Ophthalmologic conditions of AIDS patients with long-term follow-up
}

\author{
Márcia Abelin Vargas ${ }^{1}$ \\ Maria de Lourdes Veronese Rodrigues ${ }^{2}$ \\ José Fernando de Castro Fiǵueiredo ${ }^{3}$ \\ Nivaldo Vieira de Souza ${ }^{4}$
}

\section{RESUMO}

Objetivo: Avaliar as condições oftalmológicas atuais de pacientes com síndrome da imunodeficiência adquirida (SIDA), previamente avaliados por oftalmologista, levando em consideração algumas características gerais relacionada com essa doença. Métodos: Estudo observacional de 42 pacientes com SIDA, subdivididos em dois grupos: Grupo I: 8 pacientes com SIDA e diagnóstico prévio de retinite por citomegalovírus; Grupo II: 34 pacientes com SIDA sem retinite por citomegalovírus. Os dados gerais relacionados com a SIDA foram obtidos pela análise dos prontuários médicos. Resultados: A maioria dos pacientes apresentou acuidade visual no melhor olho entre logMAR 0,0 (68,3\%) e 0,1 (26,9\%). Prescrição óptica para longe beneficiou 39,4\% dos pacientes do Grupo II mas nenhum dos paciente do Grupo I. Presbiopia foi corrigida em 27,3\% no Grupo II e 12,5\% no Grupo I. Não foram encontradas manifestações oculares atuais relacionadas a SIDA em nenhum dos grupos. As alterações fundoscópicas encontradas em 10 pacientes foram todas alterações cicatriciais de retinite/ retinocoroidite, sendo $7(16,7 \%)$ pacientes pertencentes ao Grupo I e 3 $(7,1 \%)$ pacientes pertencentes ao Grupo II. Conclusão: Dez $(24,4 \%)$ pacientes apresentaram alteração visual decorrente do envelhecimento. Com exceção dos pacientes com cicatrizes prévias de retinite ou retinocoroidite, todos os outros participantes estavam em boas condições oftalmológicas e a maioria dos mesmos se encontrava em recuperação imunológica, devido ao uso da terapia anti-retroviral de alta potência.

Descritores: Síndrome de imunodeficiência adquirida; Manifestações oculares; Retinite por citomegalovírus; Terapia anti-retroviral de alta atividade

\section{INTRODUÇÃ̃O}

A infecção pelo HIV-1, quando não tratada, é marcada pelo declínio progressivo das células que compõem o sistema imune, especialmente no número de linfócitos $\mathrm{T} \mathrm{CD}_{4}^{+}$circulantes, o que, em um período variável de anos leva à morte por falência imune e infecções oportunistas ${ }^{(1)}$. A partir de meados da década de 1990, foram introduzidos esquemas terapêuticos compostos por combinações de inibidores da transcriptase reversa, nucleosídeos e não nucleosídeos e/ou inibidores de protease, com o objetivo de controlar a replicação do HIV-1 e, por conseguinte, aumentar a contagem de linfócitos $\mathrm{T} \mathrm{CD}_{4}^{+}$, reconstituindo o sistema imunológico ${ }^{(2)}$. Esses esquemas, conhecidos como terapia anti-retroviral de alta potência (HAART), resultaram em significativo aumento na sobrevida dos pacientes ${ }^{(3)}$.

$\mathrm{O}$ acometimento ocular na SIDA é potencialmente ameaçador para a 
visão, reduzindo a qualidade de vida dos pacientes se, como consequiência, a visão diminuir muito. Essas lesões podem ter aparência clínica atípica, seguir um curso incomum e, não infreqüentemente, demonstrar refratariedade ao tratamento convencional $^{(4)}$. A freqüência de acometimento ocular na SIDA varia de $21,4 \%$ a $95 \%$ dos casos, sendo menor em pacientes não-hospitalizados ${ }^{(5-7)}$ uma vez que essa freqüência relacionase com o grau de imunidade dos pacientes estudados ${ }^{(8)}$.

Desde a primeira publicação das manifestações oculares na SIDA, estudos subseqüentes tem documentado numerosas condições no olho e órbita relacionadas com a SIDA, com resultados bastante semelhantes ${ }^{(5,8-12)}$.

No hemisfério norte e no Brasil a infecção ocular mais comum em pacientes com SIDA é a retinite por citomegalovírus (RCMV), que produz deterioração progressiva da retina com marcada diminuição da acuidade visual se acometer a parte central da retina ${ }^{(8,10-12)}$. O desenvolvimento da RCMV ocorre comumente nos estágios mais tardios da SIDA, quando os pacientes apresentam imunossupressão mais acentua$\mathrm{da}^{(13)}$. Pacientes, portanto, com contagem baixa de células $\mathrm{T}$ $\mathrm{CD}_{4}^{+}\left(<50\right.$ células $\left./ \mathrm{mm}^{3}\right)$ têm risco aumentado de desenvolvê$\mathrm{la}^{(12,14)} \mathrm{e}$ estudos sugerem que aproximadamente 20 a $45 \%$ de pacientes com SIDA irão desenvolvê-la em algum momento do curso da doença ${ }^{(15-16)}$.

O diagnóstico da RCMV é clínico, pois sua aparência fundoscópica é característica ${ }^{(5,17)}$. Diferente de outras formas de retinite necrotizante, inflamação ocular marcada não é vista e o olho usualmente está calmo externamente ${ }^{(4)}$. As perdas visuais ocorrem quando há envolvimento macular ou descolamento de retina secundário, que ocorre em até $30 \%$ dos $\operatorname{casos}^{(18)}$. Atrofia óptica e edema macular cistóide podem ocorrer ${ }^{(17)}$.

Desde a introdução da HAART, tem havido um decréscimo na incidência do diagnóstico de SIDA, de infecções oportunistas, incluindo a RCMV, e da mortalidade entre os pacientes infectados com o HIV-1 ${ }^{(3)}$. Entretanto, embora a incidência da RCMV tenha declinado marcadamente, e o risco da perda visual devido à retinite $\mathrm{e}$ ao descolamento de retina tenha diminuído cerca de $30 \%$ desde o advento da HAART ${ }^{(19)}$, ela permanece a principal causa de morbidade $\operatorname{ocular}^{(20)} \mathrm{em}$ pacientes com SIDA.

Os objetivos do presente trabalho foram: 1) avaliar as modificações das condições oftalmológicas (acuidade visual, presença de lesões ativas ou complicações oculares relacionadas) de pacientes com SIDA com e sem RCMV, após um período mínimo de três anos da avaliação oftalmológica inicial, correlacionando-as com a contagem de linfócitos $\mathrm{T} \mathrm{CD}_{4}^{+}$, carga viral do HIV e uso de HAART.

\section{MÉTODOS}

Foi realizado um estudo observacional em pacientes com síndrome da imunodeficiência adquirida (SIDA), diagnosticada com base em manifestações clínicas e laboratoriais ${ }^{(21)}$, sobreviventes de estudo anterior ${ }^{(22)}$.
Desde a fase inicial, esta pesquisa foi aprovada pela Comissão de Ética do HCFMRP (Processo HCRP nํ1525/98).

Os pacientes participantes deste estudo foram selecionados a partir do banco de dados do estudo, cujos dados foram colhidos até março de $1999^{(22)}$, do qual participaram 44 pacientes com SIDA e RCMV e 80 pacientes com SIDA sem RCMV.

Após levantamento, através do Registro de Óbitos e da Vigilância Epidemiológica do HCFMRP, constatou-se que dos 44 pacientes com SIDA e RCMV, 32 (72,7\%) já haviam falecido e $1(2,3 \%)$ paciente não foi localizado, e que dos 80 pacientes com SIDA sem RCMV, 33 (41,3\%) já haviam ido a óbito, não tendo sido localizados $4(5,0 \%)$ pacientes. Portanto, foram inicialmente convidados a participarem do presente estudo 11 pacientes com SIDA e RCMV e 43 pacientes com SIDA e sem RCMV.

Assim, a população final do estudo foi constituída por 8 pacientes com SIDA e RCMV (Grupo I) e por 34 pacientes com SIDA sem RCMV (Grupo II), sobreviventes do estudo anterior $^{(22)}$ e que atenderam à convocação e concordaram em participarem deste estudo.

Os dados demográficos, clínicos e laboratoriais foram obtidos através da análise dos prontuários médicos, sendo estes os mais próximos da data do exame oftalmológico atual.

As características gerais dos dois grupos estão relacionadas no quadro 1.

Após a anamnese, o exame oftalmológico constou de medida e registro da acuidade visual (com a melhor correção óptica encontrada), biomicroscopia e oftalmoscopia binocular indireta sob midríase.

A medida da acuidade visual foi realizada em cada olho, separadamente, utilizando-se tabela com optotipos de Snellen disposta a seis metros de distância do paciente. Nos pacientes que não enxergaram o valor 0,1 da tabela, a visão foi testada através de outros métodos, com graduação visual regressiva: contagem de dedos do examinador a distâncias variáveis; percepção de movimentos da mão do examinador próxima ao paciente; percepção da luz de uma lanterna; ou ausência de percepção luminosa - nihil. Os dados da acuidade visual foram posteriormente convertidos para escala logarítmica, de acordo com a tabela de conversão para escala logarítmica do mínimo ângulo de resolução - $\log \mathrm{MAR}^{(23)}$.

\section{RESULTADOS}

A maioria dos pacientes apresentou acuidade visual (AV) no melhor olho entre logMAR $0,0(68,3 \%)$ e $\log$ MAR 0,1 $(26,9 \%)$ (Tabela 1). Uma paciente não pode informar sua acuidade visual pelas más condições clínicas.

No Grupo I, dos oito pacientes examinados, sete pacientes apresentaram AV no melhor olho AV igual ou maior que $\log$ MAR 0,1 (logMAR 0,0 (50\%) e logMAR 0,1 (37,5\%)), Do registro de dados do estudo anterior ${ }^{(22)}$ obteve-se o resultado da mensuração da AV em cinco dos pacientes com SIDA e RCMV que foram reavaliados. Comparando-se a AV anterior e 


\begin{tabular}{|c|c|c|c|}
\hline Parâmetro & Grupo I $(\mathrm{N}=8)$ & Grupo II (N=34) & p \\
\hline Gênero & $\begin{aligned} M & =6 \\
F & =2\end{aligned}$ & $\begin{aligned} M & =23 \\
F & =11\end{aligned}$ & 1,0 \\
\hline Idade & $\begin{aligned} \text { Mediana } & =38 \text { anos } \\
M n & =31 \text { anos } \\
M x & =41 \text { anos }\end{aligned}$ & $\begin{aligned} \text { Mediana } & =38,5 \text { anos } \\
M n & =29,0 \text { anos } \\
M x & =56,0 \text { anos }\end{aligned}$ & 0,40 \\
\hline Uso de HAART & $\begin{aligned} \operatorname{Sim} & =8 \\
\text { Não } & =0\end{aligned}$ & $\begin{aligned} \operatorname{Sim} & =28 \\
\text { Não } & =6\end{aligned}$ & 0,57 \\
\hline Tempo de seguimento & $\begin{aligned} \text { Mediana } & =77 \text { meses } \\
M n & =54 \text { meses } \\
M x & =89 \text { meses }\end{aligned}$ & $\begin{aligned} \text { Mediana } & =68 \text { meses } \\
M n & =50 \text { meses } \\
M x & =144 \text { meses }\end{aligned}$ & 0,79 \\
\hline Número de infecções oportunistas & $\begin{aligned} \text { Mediana } & =3,5 \\
M n & =1 \\
M x & =7\end{aligned}$ & $\begin{aligned} \text { Mediana } & =2,5 \\
M n & =0 \\
M x & =6\end{aligned}$ & 0,09 \\
\hline Número de linfócitos $\mathrm{T} \mathrm{CD}_{4}{ }^{+} / \mathrm{mm}^{3}$ & $\begin{aligned} \text { Mediana } & =366,5 \\
M n & =24 \\
M x & =1119\end{aligned}$ & $\begin{aligned} \text { Mediana } & =277 \\
M n & =16 \\
M x & =802\end{aligned}$ & 0,48 \\
\hline Log da viremia do HIV-1 detectável & $\begin{aligned} \text { Mediana } & =3,61 \\
\mathrm{Mn} & =3,09 \\
\mathrm{Mx} & =4,41\end{aligned}$ & $\begin{aligned} \text { Mediana } & =4,41 \\
M n & =1,95 \\
M x & =6,24\end{aligned}$ & 0,67 \\
\hline № de pacientes com viremia do HIV-1 indetectável & $3 / 8$ & $12 / 31$ & 1,0 \\
\hline
\end{tabular}

a atual, observa-se que $3(60 \%)$ dos cinco pacientes apresentaram piora em um dos olhos, durante o período, devido às seqüelas da RCMV (Tabela 2).

No Grupo II, 33 pacientes dos 34 examinados apresentaram AV igual ou maior que logMAR 0,1 em pelo menos um dos olhos (logMAR 0,0 (72,7\%) e logMAR 0,1 (24,2\%)). Uma paciente não pôde informar sua AV pelas más condições clínicas.

Além de realizarem o exame oftalmológico proposto na metodologia, $19(57,6 \%)$ dos $33(97,1 \%)$ pacientes do Grupo II receberam prescrição óptica para corrigir ametropias variadas, $13(39,4 \%)$ pacientes recebendo auxílio para melhora da AV para longe e $9(27,3 \%)$ pacientes apresentando presbiopia. No Grupo I, dos oito pacientes examinados, apenas 1 (12,5\%) paciente beneficiou-se de correção óptica para presbiopia.

No exame biomicroscópico do Grupo I foram observadas alterações em $3(37,5 \%)$ dos 8 pacientes examinados, sendo elas: catarata unilateral (2 pacientes), sinéquias posteriores ( 2

\begin{tabular}{|cc|}
\hline $\begin{array}{c}\text { Tabela 1. Distribuição da acuidade visual (LogMAR), no olho de } \\
\text { melhor visão, obtida em 41 pacientes com SIDA - Hospital das }\end{array}$ \\
Clínicas da Faculdade de Medicina de Ribeirão Preto - 2003 \\
Valor de AV no melhor olho & Número de pacientes \\
0,0 & 28 \\
0,1 & 11 \\
0,2 & 0 \\
0,3 & 1 \\
0,4 & 0 \\
0,5 & 1 \\
0,7 & 0 \\
0,8 & 0 \\
1,0 & 0 \\
\hline
\end{tabular}

pacientes), atrofia de íris (1 paciente) e pterígio (1 paciente). No Grupo II, $14(42,4 \%)$ dos 33 pacientes examinados apresentaram alterações oculares externas: blefarite seborreica (6 pacientes), pterígio (6 pacientes) e conjuntivite viral aguda (2 pacientes). No total dos 41 pacientes nos quais realizou-se a biomicroscopia, $24(58,5 \%)$ não apresentaram alterações detectáveis nesse exame.

O exame do fundo de olho foi viável em 14 olhos dos oito pacientes do Grupo I; destes, $11(78,6 \%)$ apresentaram cicatrizes de RCMV e 2 (12,5\%) não puderam ser examinados devido à presença de catarata total secundária (2 pacientes). Nesse mesmo grupo, $3(37,5 \%)$ pacientes apresentaram palidez de nervo óptico e $2(25 \%)$ apresentaram cicatriz de RCMV macular. $\mathrm{O}$ acometimento binocular da RCMV foi observado em $5(62,5 \%)$ pacientes.

\begin{tabular}{|c|c|c|c|c|}
\hline \multirow[t]{2}{*}{ Paciente } & \multicolumn{2}{|c|}{2000} & \multicolumn{2}{|c|}{2003} \\
\hline & AV-OD & AV-OE & AV-OD & AV-OE \\
\hline 1 & - & - & 0,1 & 0,3 \\
\hline 2 & 0,2 & 0,0 & 0,5 & 0,0 \\
\hline 3 & - & - & 0,4 & MM \\
\hline 4 & 0,1 & 0,2 & 0,1 & MM \\
\hline 5 & 0,3 & 1,0 & 0,0 & nihil \\
\hline 6 & 0,7 & 0,0 & 0,7 & 0,0 \\
\hline 7 & 0,1 & 0,3 & 0,1 & 0,3 \\
\hline 8 & - & - & 0,8 & 0,0 \\
\hline
\end{tabular}


No grupo II, dos 68 olhos submetidos ao exame fundoscópico, $2(2,9 \%)$ apresentaram cicatrizes compatíveis com retinocoroidite por toxoplasmose e $2(2,9 \%)$ apresentaram cicatrizes retinianas de etiologia incerta, sendo esses últimos pertencentes a uma mesma paciente.

Os dados evolutivos da contagem de linfóctos $\mathrm{T} \mathrm{CD}_{4}^{+}$e do logaritmo da carga viral do HIV-1, nos pacientes do Grupo I, no intervalo entre as avaliações oftalmológicas, estão apresentados na tabela 3 .

\section{DISCUSSÃO}

Os Grupos I e II foram semelhantes no que diz respeito ao gênero, à idade e às características clínicas e laboratoriais da SIDA. Trinta e seis pacientes estava em uso de HAART (85,7\%), 30 pacientes apresentavam contagem de linfócitos T $\mathrm{CD}_{4}^{+} \geq 100$ células $/ \mathrm{mm}^{3}(71,4 \%)$ e 34 pacientes tinham a contagem da carga viral $<50.000$ cópias $/ \mathrm{ml}(\log =4,69)(80,9 \%)$. Esses três últimos dados os excluem de estarem em risco de desenvolvimento de RCMV, pelos critérios usados por Rodrigues ${ }^{(22)}$.

A mediana do tempo de diagnóstico de SIDA neste estudo foi de 77 meses (mínimo de 54 meses; máximo de 89 meses) para o Grupo I e de 68 meses (mínimo de 50 meses; máximo de 144 meses) para o Grupo II.

Nenhum paciente examinado apresentou alterações oculares atuais relacionadas a SIDA. Como já mencionado, a maioria dos pacientes encontrava-se em boas condições imunológicas. Sabe-se que as alterações oculares relacionadas a SIDA manifestam-se, principalmente, em fases mais avançadas da infecção pelo HIV-1, quando a contagem de células $\mathrm{T} \mathrm{CD}_{4}^{+}$é menor que 50 células $/ \mathrm{mm}^{(3,14)}$. Mesmo os pacientes que apresentavam contagem de células $\mathrm{TCD}_{4}{ }^{+}$menor que 50 células $/ \mathrm{mm}^{3}$ (1 no Grupo I e 6 no Grupo II), não apresentavam alterações oculares ativas relacionadas a SIDA por ocasião do último exame oftalmológico, sendo que desses, apenas um, do Grupo II, não estava em uso da HAART.

Entre os 42 pacientes examinados, a maioria (39 pacientes)

\begin{tabular}{|c|c|c|c|c|}
\hline \multirow[t]{2}{*}{ Paciente } & \multicolumn{2}{|c|}{$\begin{array}{l}\text { Contagem de } \\
\text { linfócitos } \mathrm{TCD}_{4}^{+}\end{array}$} & \multicolumn{2}{|c|}{$\begin{array}{l}\text { Logaritmo da } \\
\text { carga viral }\end{array}$} \\
\hline & 2000 & 2003 & 2000 & 2003 \\
\hline 1 & 24 & 92 & 4,83 & 3,09 \\
\hline 2 & 7 & 24 & 4,70 & 4,41 \\
\hline 3 & 210 & 418 & - & 3,61 \\
\hline 4 & 42 & 512 & 4,71 & 3,31 \\
\hline 5 & 6 & 157 & 5,68 & 3,69 \\
\hline 6 & 108 & 1119 & 4,49 & ALD \\
\hline 7 & 13 & 315 & 6,39 & ALD \\
\hline 8 & 11 & 434 & 5,60 & ALD \\
\hline \multicolumn{5}{|c|}{ ALD: abaixo do limite de detecção } \\
\hline
\end{tabular}

apresentou acuidade visual no melhor olho entre 1,0 (logMAR $0,0)(68,3 \%)$ e $0,8(\log$ MAR 0,1$)(26,2 \%)$. Uma paciente do Grupo II não pode informar sua acuidade visual pelas más condições clínicas. No Grupo I, entre os 8 pacientes examinados, a maioria apresentou acuidade visual no melhor olho entre 1,0 (logMAR $0,0)(50 \%)$ e $0,8(\log$ MAR 0,1$)(37,5 \%)$, sendo que sete pacientes $(87,5 \%)$ apresentavam acuidade visual igual ou maior que 0,8 (logMAR 0,1$)$ em pelo menos um dos olhos, apesar da sequiela deixada pela RCMV prévia. A combinação da HAART e das eficazes drogas anti-CMV têm melhorado o prognóstico visual dos pacientes com RCMV, e têm reduzido muito o risco do desenvolvimento bilateral de perdas visuais importantes ${ }^{(24)}$. O risco de perda visual devido à retinite e ao descolamento de retina diminuiu cerca de $30 \%$ desde o advento da HAART ${ }^{(19)}$. O fato de todos os pacientes do Grupo I estarem em uso de HAART e terem feito uso de ganciclovir EV permite a mesma conclusão dos estudos supracitados.

Apenas os pacientes do Grupo II se beneficiaram da correção óptica da ametropia encontrada, pois os pacientes com baixa de acuidade visual em algum dos olhos, pertencentes ao Grupo I, apresentavam perda visual irrecuperável devido às seqüelas da RCMV.

A prevalência de presbiopia funcional tem uma tendência ao aumento com a idade, $6,1 \%$ das pessoas na faixa etária de 30 a 34 anos apresentaram dificuldade visual de perto, ao passo que $59,9 \%$ dos indivíduos no grupo dos 70 anos ou mais tiveram a mesma condição, em estudo de prevalência de deficiência visual de perto realizado em 3.007 adultos acima de 30 anos em Pelotas, Rio Grande do $\mathrm{Sul}^{(25)}$.

No presente estudo, os pacientes com SIDA do Grupo I apresentavam idades variando de 31 a 41 anos (mediana $=38$ anos) e os pacientes do Grupo II de 29 a 56 anos (mediana= 38,5 anos). Presbiopia foi a queixa de $1(12,5 \%)$ paciente do Grupo I com 37 anos de idade e de $9(27,3 \%)$ pacientes do Grupo II, com idades que variaram de 39 a 56 anos. Nos dois grupos, a menor idade em que a presbiopia foi encontrada, foi em pacientes com SIDA do sexo feminino. A noção de que o aparecimento de presbiopia é mais precoce no sexo feminino foi suportada por Duarte et al. ${ }^{(25)}$ que verificaram que as mulheres tiveram um risco global de $22 \%$ a mais de serem acometidas que os homens.

O perfil das alterações oculares relacionadas a SIDA sofreu alterações desde a introdução da HAART, pois os pacientes, outrora imunodeprimidos, experimentam, na maioria das vezes, uma surpreendente recuperação imunológica mas, infelizmente, complicações oculares relacionadas a SIDA continuam a ocorrer, especialmente as infecções oportunistas, que muitas vezes deixam como seqüela a baixa visão ou a cegueira. Apesar dos esforços para conter-se a evolução fatal da SIDA, tem-se observado a ocorrência de resistência viral aos produtos disponíveis ${ }^{(26)}$.

Devido a essas constatações, o cuidadoso monitoramento de pacientes com SIDA se faz necessário, pois com o aumento da sobrevida desses pacientes, a importância da manutenção de uma boa visão, mantendo a qualidade de vida, é uma prioridade. 


\section{CONCLUSÕES}

A maioria dos pacientes avaliados apresentou acuidade visual no melhor olho entre $\log$ MAR $0,0(68,3 \%)$ e $\log$ MAR 0,1 $(26,9 \%)$. No intervalo de tempo entre as duas avaliações oftalmológicas, como consequiência da HAART, houve melhora significativa dos parâmetros imunológicos e virológicos dos pacientes. Em razão disso, os pacientes que não tinham lesões prévias apresentaram condições oftalmológicas semelhantes a de pacientes imunocompetentes. Nenhum dos oito pacientes com retinite prévia por citomegalovírus apresentou lesão em atividade na segunda avaliação.

\section{ABSTRACT}

Purpose: To evaluate the ophthalmologic conditions of patients with AIDS, with long-term follow-up, previously evaluated by an ophthalmologist, considering general conditions related with AIDS. Methods: Observational study of 42 patients with AIDS divided into two groups: Group I: 8 patients with previous AIDS-related cytomegalovirus retinitis, Group II: 34 patients with AIDS without cytomegalovirus retinitis. Each patient had been submitted to one ophthalmologic examination. General data of the patients were obtained from the medical records. Results: The majority of the patients presented visual acuity in the best eye between $\log$ MAR 0.0 $(68.3 \%)$ and $0.1(26.9 \%)$. Optic prescription for refractive errors benefited $39.4 \%$ of the patients in Group II but none of the patients in Group I. Presbyopia was corrected in $27.3 \%$ of Group II and in $12.5 \%$ of Group I patients. No current ocular AIDS-related manifestations were detected in any group. Ocular posterior segment alterations, all of them consisting of retinitis / retinochoroiditis scars, were found in 10 patients, 7 $(16.7 \%)$ belonging to Group I and 3 (7.1\%) to Group II. Conclusion: Ten $(24.4 \%)$ patients presented visual alteration due to age. Except for the patients presenting previous retinitis and retinochoroiditis, all the other patients were in good ophthalmic conditions and most of them were in immunologic recovery due to the use of highly active antiretroviral therapy.

Keywords: Acquired immunodeficiency syndrome; Eye manifestations; Cytomegalovirus retinitis; Antiretroviral therapy, highly active

\section{REFERÊNCIAS}

1. Haynes BF, Pantaleo G, Fauci AS. Toward an understanding of the correlates of protective immunity to HIV infection. Science. 1996;271(5247):324-8. Review.

2. Ho D. Plenary Abstract Th19*. In: XI International Conference on AIDS, Vancouver, 1996; July,7-12.

3. Palella FJ Jr, Delaney KM, Moorman AC, Loveless MO, Fuhrer J, Satten GA, et al. Declining morbidity and mortality among patients with advanced human immunodeficiency virus infection. HIV Outpatient Study Investigators. N Engl J Med. 1998;338(13):853-60.

4. Ah-Fat FG, Batterbury M. Ophthalmic complications of HIV/AIDS. Postgrad Med J. 1996;72(854):725-30. Review.

5. Palestine AG, Rodrigues MM, Macher AM, Chan CC, Lane HC, Fauci AS, et al. Ophthalmic involvement in acquired immunodeficiency syndrome. Ophthalmology. 1984;91(9):1092-9.

6. Humphry RC, Weber JN, Marsh RJ. Ophthalmic findings in a group of ambulatory patients infected by human immunodeficiency virus (HIV): a prospective study. Br J Ophthalmol. 1987;71(8):565-9.

7. Pinheiro SRAA, Oréfice F, Greco DB, Antunes CMF. Freqüência das manifestações oculares nos pacientes com sorologia positiva para o HIV ou de risco para a infecção na cidade de Belo Horizonte de 1990 a 1992: parte I. Rev Bras Oftalmol. 1996;55(1):17-23.

8. Curi ALL, Muralha Neto A, Muranaka E, Muralha L, Vianna RNG. Estudo epidemiológico das alterações oftalmológicas na Síndrome da Imunodeficiência Adquirida. Rev Bras Oftalmol. 1999;58(3):223-6.

9. Holland GN, Gottlieb MS, Yee RD, Schanker HM, Pettit TH. Ocular disorders associated with a new severe acquired cellular immunodeficiency syndrome. Am J Ophthalmol. 1982;93(4):393-402.

10. Jabs DA, Green WR, Fox R, Polk FB, Bartlett JG. Ocular manifestations of acquired immune deficiency syndrome. Ophthalmology. 1989;96(7):1092-9.

11. Muccioli C, Belfort Júnior R, Lottenberg C, Lima J, Santos P, Kim M, et al. Achados oftalmológicos em AIDS: avaliação de 445 casos atendidos em um ano. Rev Assoc Med Bras (1992). 1994;40(3):155-8.

12. Dualiby PT, Suleiman JMAH. Achados oculares em AIDS no Instituto de Infectologia Emílio Ribas antes da introdução das drogas inibidoras de protease. Rev Bras Oftalmol. 1999;58(5):383-7.

13. Holland GN. Acquired immunodeficiency syndrome and ophthalmology: the first decade. Am J Ophthalmol. 1992;114(1):86-95.

14. Kuppermann BD, Petty JG, Richman DD, Mathews WC, Fullerton SC, Rickman LS, Freeman WR. Correlation between CD4+ counts and prevalence of cytomegalovirus retinitis and human immunodeficiency virus-related noninfectious retinal vasculopathy in patients with acquired immunodeficiency syndrome. Am J Ophthalmol. 1993;115(5):575-82.

15. Schrier RD, Freeman WR, Wiley CA, McCutchan JA. Immune predispositions for cytomegalovirus retinitis in AIDS. The HNRC Group. J Clin Invest. 1995;95(4):1741-46

16. Jabs DA, Quinn TC. Acquired immunodeficiency syndrome (AIDS). In: Pepose JS, Holland GN, Wilhelmus KR, editors. Ocular infection and immunity. St. Louis: Mosby; 1996. p.289-304.

17. Muccioli C, Belfort Jr R. Retinite por citomegalovírus. In: Muccioli C, Belfort Jr R. Manifestações oculares da aids: atlas \& texto. Rio de Janeiro: Cultura Médica; 1999. p.56-70.

18. Muralha NA, Curi ALL. AIDS Ocular - Parte II. In: Oréfice F. Uveíte clínica e cirúrgica: texto e atlas. Rio de Janeiro: Cultura Médica; 2000. v.1, p.541-54.

19. Holbrook JT, Jabs DA, Weinberg DV, Lewis RA, Davis MD, Friedberg D. Studies of Ocular Complications of AIDS (SOCA) Research Group. Visual loss in patients with cytomegalovirus retinitis and acquired immunodeficiency syndrome before widespread availability of highly active antiretroviral therapy. Arch Ophthalmol. 2003;121(1):99-107.

20. Jabs DA. AIDS and ophthalmology in 2004. Arch Ophthalmol. 2004;122(7): 1040-2.

21. 1993 revised classification system for HIV infection and expanded surveillance case definition for AIDS among adolescents and adults. MMWR Recomm Rep. 1992;42(RR-17):1-19.

22. Rodrigues MLV. Análise de antígenos e alelos de histocompatibilidade de classe I e II, em pacientes com aids e com aids e retinite por citomegalovírus [tese]. Ribeirão Preto - Faculdade de Medicina de Ribeirão Preto da Universidade de São Paulo; 2000.

23. Bailey IL, Lovie JE. New design principles for visual acuity letter charts. Am J Optom Physiol Opt. 1976;53(11):740-5.

24. Kestelyn PG, Cunningham ET Jr. HIV/AIDS and blindness. Bull World Health Organ. 2001;79(3):208-13.

25. Duarte WR, Barros AJD, Dias-da-Costa JS, Cattan JM. Prevalência de deficiência visual de perto e fatores associados: um estudo de base populacional. Cad Saúde Pública. 2003;19(2):551-9.

26. Kantor R, Shafer RW, Follansbee S, Taylor J, Shilane D, Hurley L, et al. Evolution of resistance to drugs in HIV-1 infected patients failing antiretroviral therapy. AIDS. 2004;18(11):1503-11. 